\title{
Complete hydatidiform mole retaining a chromosome 11 of maternal origin: molecular genetic analysis of a case
}

\author{
Rosemary A Fisher ${ }^{1}$, Marisa R Nucci ${ }^{2}$, Harshwardhan M Thaker ${ }^{3}$, \\ Stanislawa Weremowicz ${ }^{2}$, David R Genest ${ }^{2}$ and Diego H Castrillon ${ }^{2, *}$ \\ ${ }^{1}$ Department of Cancer Medicine, Faculty of Medicine, Imperial College London, Charing Cross Hospital, \\ London, UK; ${ }^{2}$ Division of Women's and Perinatal Pathology, Department of Pathology, Brigham and Women's \\ Hospital and Harvard Medical School, Boston, MA, USA and ${ }^{3}$ Department of Pathology, Columbia University \\ College of Physicians \& Surgeons, New York, NY, USA
}

\begin{abstract}
Hydatidiform moles are pregnancies characterized by abnormal development of both embryonic and extraembryonic tissues and are associated with the misexpression of imprinted genes. The vast majority of complete hydatidiform moles are diploid and androgenetic, whereas partial hydatidiform moles are triploid, with an extra set of chromosomes of paternal origin. Here, we present an unusual complete mole that showed strong expression of two imprinted, maternally transcribed genes, CDKN1C (encoding $\mathrm{p} 57^{\mathrm{KIP} 2}$ ) and PHLDA2 (TSSC3/ $I P L)$, both part of a large imprinted gene domain on chromosome 11. Using microsatellite genotyping and fluorescent in situ hybridization, we show that this paradoxical gene expression was due to retention of a maternal copy of chromosome 11 in addition to the two paternal copies normally present in complete moles. These findings demonstrate that, despite being predominantly androgenetic, some complete moles contain small amounts of DNA of maternal origin. Furthermore, these results provide an explanation for rare false negatives that can arise when $\mathrm{p} 57^{\mathrm{KIP} 2}$ is used as a diagnostic marker for complete moles.

Modern Pathology (2004) 17, 1155-1160. doi:10.1038/modpathol.3800175
\end{abstract}

Keywords: genomic imprinting; hydatidiform mole; p57 ${ }^{\mathrm{KIP} 2}$; CDKN1C; PHLDA2; IPL; trisomy 11

Hydatidiform mole is a gestation characterized by abnormal development of both fetus and trophoblast. $^{1}$ The majority of hydatidiform moles are associated with an excess of paternal to maternal genomes and are likely to result from the abnormal expression of imprinted genes. Hydatidiform moles are subclassified on the basis of histopathology, clinical features, and ploidy as partial or complete. Partial moles arise from the fertilization of a normal egg by two spermatozoa (dispermy) or in rare cases a diploid spermatozoon, resulting in a triploid gestation with one maternal and two paternal sets of chromosomes. $^{2}$ The great majority of complete moles are entirely androgenetic, lacking nuclear DNA of maternal origin. Complete moles originate by dispermy (XX or XY) or more frequently mono-

Correspondence: Dr DH Castrillon, MD, PhD, Department of Pathology, Laboratory of Molecular Pathology, University of Texas Southwestern Medical Center, Dallas, TX 75390-9072, USA.

E-mail: Diego.Castrillon@UTSouthwestern.edu

${ }^{*}$ Current address: Department of Pathology, University of Texas Southwestern Medical Center, Dallas, TX, USA.

Received 13 February 2004; revised and accepted 8 April 2004 spermy (always XX), where the sperm pronucleus replicates prior to the first cleavage division. The mechanisms underlying the failure of the egg to contribute a normal complement of chromosomes to the zygote remain obscure, but might relate to errors during meiosis.

We have previously demonstrated that $\mathrm{p} 57^{\mathrm{KIP} 2}$, the product of the imprinted maternally expressed gene $C D K N 1 C$, is misexpressed in complete moles and that this misexpression can be readily detected by immunohistochemistry on paraffin-embedded formalin-fixed tissue to aid in the diagnosis of complete moles. ${ }^{3-5}$ CDKN1C is part of a large domain on chromosome 11p15.5 that contains other imprinted genes such as PHLDA2 (IPL/TSSC3) ${ }^{6}$ and $I G F 2 .^{7}$ Consistent with the fact that $C D K N 1 C$ is preferentially expressed from its maternal copy in most cell types, ${ }^{8}$ the ${ }^{2} 57^{\mathrm{KIP} 2}$ protein is readily detectable in placental villi (cytotrophoblast and villous mesenchyme) of normal gestations and partial moles but is absent in most villous cells in complete moles, which lack nuclear DNA of maternal origin. ${ }^{3}$ In the course of these studies, we identified an unusual case of a complete mole that 
showed strong expression of p57 $7^{\mathrm{KI} 2}$ in placental villi in a normal (ie, noncomplete mole like) pattern. The aberrant pattern of $\mathrm{p} 57^{\mathrm{KIP} 2}$ expression in this complete mole has been previously reported. ${ }^{3}$ Here, we present a molecular genetic analysis of this case revealing that the mechanism underlying the aberrant pattern of p57 ${ }^{\mathrm{KIP} 2}$ expression was retention of a maternal copy of chromosome 11 encoding the CDKN1C gene.

\section{Materials and methods}

\section{Case History}

The patient was a 31-year-old $\mathrm{G}_{3} \mathrm{P}_{1}$ with no prior molar gestations. The current pregnancy was terminated at 10 weeks due to an abnormal ultrasound consistent with hydatidiform mole. She underwent spontaneous remission (without chemotherapy) within 1 year, and a subsequent pregnancy was successfully carried to term. Flow cytometry performed on fresh villous tissue returned a diploid result, consistent with the diagnosis of complete mole. Review of all slides revealed histopathologic features indicative of complete mole, including circumferential trophoblast hyperplasia and diffuse villous cavitation. However, immunohistochemistry for $\mathrm{p} 57^{\mathrm{KIP} 2}$ showed uniformly strong expression in cytotrophoblast, villous mesenchyme, and extravillous trophoblast, the typical pattern of noncomplete mole gestations, as previously reported, ${ }^{3}$ and shown in Figure 1.

\section{Immunohistochemistry}

A mouse monoclonal antibody against the human p57 ${ }^{\text {KIP2 }}$ protein from NeoMarkers/Lab Vision Corporation was used (47790 Westinghouse Dr, Fremont, CA 94539, USA). We employed a ready-to-use preparation without dilution (Catalog \#MS-1062-R7) or a concentrated preparation (Cat \#MS-1062-P1) at 1:200 dilution with similar results. Antigen retrieval on archival, paraffin-embedded formalin-fixed tissue was performed at $93^{\circ}$ in $10 \mathrm{mM}$ sodium citrate buffer pH 6.0 for $30 \mathrm{~min}$ with a 10-min cooldown. The detection system was Envision (Dako Corporation, 6392 Via Real, Carpinteria, CA 93013, USA) with diaminobenzidine as the chromogen. Immunohistochemistry for PHLDA2 was performed on paraffin-embedded formalin-fixed sections of molar tissue with an affinity-purified polyclonal antiPHLDA2 antibody (hIPL-C134) raised against a synthetic peptide (CPSEPSEPSRPSPQPKPRTP) whose amino-acid sequence was derived from the C-terminal portion of PHLDA2. ${ }^{9}$ This antibody was used at a 1:6000 dilution; antigen retrieval was carried out in $1 \mathrm{mM}$ EDTA by boiling slides in a $1000 \mathrm{~W}$ microwave oven for $8 \mathrm{~min}$ at $100 \%$ power followed by $15 \mathrm{~min}$ at $30 \%$ power. Slides were counterstained with hematoxylin.
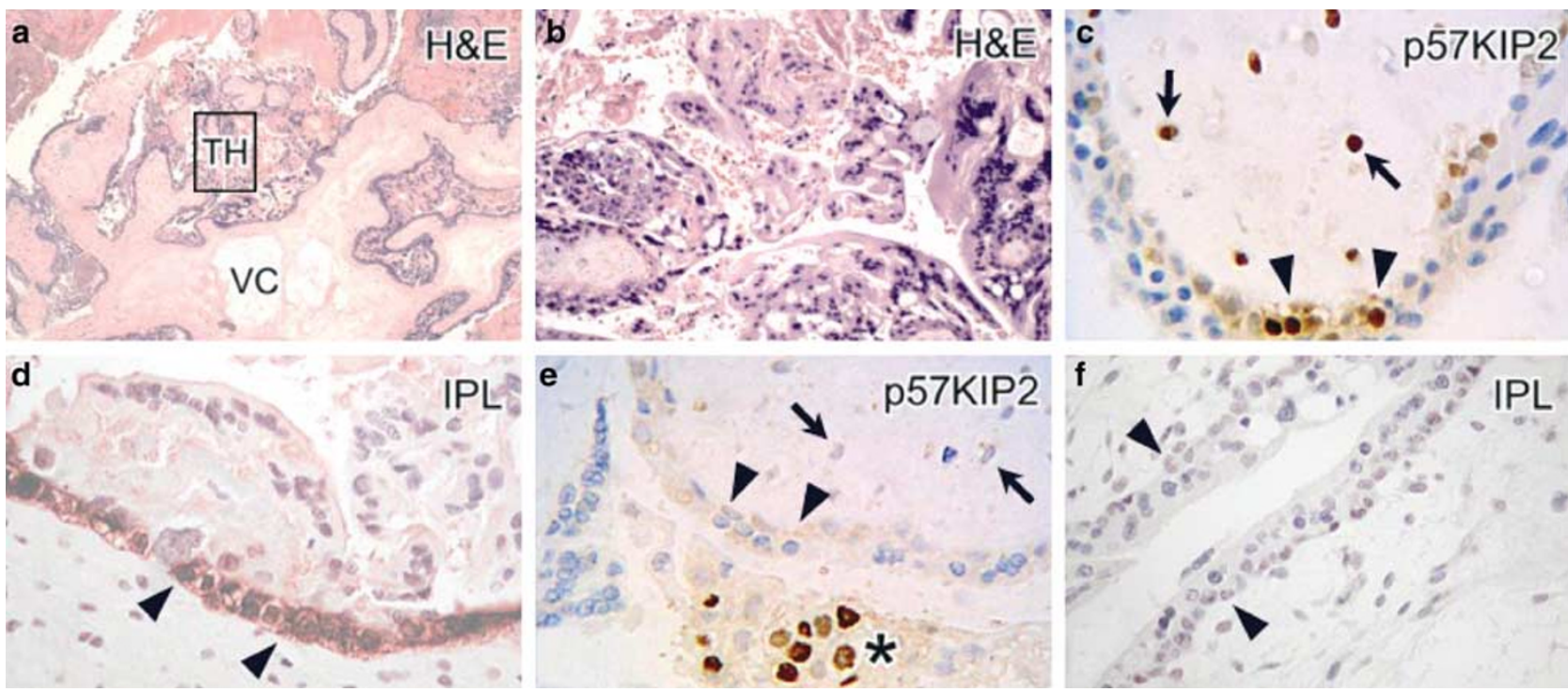

Figure 1 Case report of an unusual p57 ${ }^{\mathrm{KIP} 2}$ and PHLDA2-positive complete mole. (a,b) Hematoxylin and eosin staining showing histologic features consistent with complete mole. (a) Low magnification showing typical complete mole features including placental villi with irregular architecture, edema with true villous cavitation (VC) and significant trophoblast hyperplasia (TH). (b) Higher magnification of boxed inset from panel (a) showing significant cyto- and syncitiotrophoblast hyperplasia. (c, d) Immunostaining of this case showing strong expression of products of two maternally expressed genes that are part of the chromosome 11 imprinted domain. (c) Strong p57 $7^{\mathrm{KI} 2}$ expression in cytotrophoblast (arrowhead) and villous mesenchyme (arrow). (d) Strong PHLDA2 (IPL) expression in cytotrophoblast (arrowheads). (e,f) Example of a typical complete mole showing lack of expression of these maternally expressed gene products. (e) p57 $57^{\mathrm{KIP} 2}$, (f) PHLDA2 (IPL). For e-f, arrowheads indicate cytotrophoblast, arrows indicate villous mesenchymal nuclei, and the asterisk marks an intervillous trophoblast island (internal positive control). 


\section{Microsatellite Genotyping}

For microsatellite analysis, DNA was prepared from the complete mole and the patient tissue using microdissected villous and decidual (endometrial) tissue from the same sections. DNA from the complete mole and the patient was genotyped for a series of 12 polymorphic microsatellite markers (D11S2071; TH; D11S922; D11S2345; D11S4149; D11S1999; D11S875; D11S4116; D11S569; D11S899; D11S904; D11S907) on chromosome 11p and 5 (D11S916; D11S35; D11S925; D11S968; D11S910) on $11 q$ as previously described. ${ }^{10}$

\section{Fluorescent In Situ Hybridization}

To confirm ploidy of the molar tissue, centromeric probes for chromosomes X, Y, 8 and 18 were hybridized to interphase nuclei in suspension as previously described. ${ }^{11}$ To determine ploidy of the CDKN1C region, fluorescent in situ hybridization (FISH) was performed using a BAC probe spanning the $C D K N 1 C$ locus. BAC RP11-555F1, which contains all $C D K N 1 C$ coding sequences, was obtained (RPCI-11 human BAC library, Research Genetics), confirmed by end-sequencing, and labeled using the Random Primer system with biotin-conjugated deoxynucleotides (Gibco-BRL). Sections of tissue ( $5 \mu \mathrm{m})$ cut onto coated slides were deparaffinized in xylene and dehydrated in sequential alcohol treatments. Microwave antigen retrieval was performed in $100 \mathrm{mM}$ Tris-base, $50 \mathrm{mM}$ EDTA, pH 7.0 at $92.8^{\circ} \mathrm{C}$ for $30 \mathrm{~min}$. The slides were treated with proteinase $\mathrm{K}$ for $20 \mathrm{~min}$, washed in phosphate-buffered saline, dehydrated in sequential alcohol treatments and then incubated at $37^{\circ} \mathrm{C}$ with the DNA probe for $48 \mathrm{~h}$ following codenaturation at $94^{\circ} \mathrm{C}$ for $5 \mathrm{~min}$. The labeled probe was detected by 30 -min room temperature incubation with conjugated avidin (Alexafluor 594; Molecular Probes, Eugene, OR, USA). Following washing with PBS and staining with DAPI, the slides were coverslipped for viewing by fluorescence microscopy.

Single cell suspensions to determine ploidy for other chromosome 11 regions (centromere and $\mathrm{q}$ arm) were prepared as described $^{11}$ using $50 \mu \mathrm{m}$ sections cut from a block of formalin-fixed paraffin-embedded molar tissue. FISH on interphase nuclei was performed with chromosome 11 specific DNA probes: CEP11 at 1p11.1-q11.1 (green) and Cyclin D at 11q13 (red) (Vysis, Inc.). Nuclei were counterstained with DAPI. Slides were examined using a fluorescence microscope. Images were captured using CytoVysion Imaging System (Applied Imaging, Pittsburgh, PA, USA).

\section{Results}

The unexpected expression of $\mathrm{p} 57^{\mathrm{KIP} 2}$ in an otherwise typical complete mole (Figure 1a-c) prompted an investigation of its basis. Immunostaining for PHLDA2, the product of a second maternally expressed gene located within the same imprinted domain on 11p15.5, also showed aberrant patterns of expression in this complete mole, being strongly expressed in cytotrophoblast (Figure 1d). PHLDA2 is normally expressed specifically in the cytotrophoblast of normal gestations and partial moles. Like p57 ${ }^{\mathrm{KIP} 2}$, PHLDA2 is undetectable in these cells in most complete moles (Figure 1e,f) as expected given the monoallelic (maternal) expression of PHLDA2 and the absence of maternal DNA in complete moles.

Flow cytometry, using fresh tissue collected at the time of diagnosis, returned a diploid result. To confirm this finding, FISH was performed using centromeric probes for chromosomes X, Y, 8, and 18. These studies showed that this complete mole was $\mathrm{XX}$ and diploid, excluding the possibility that it represented a partial mole (Figure 2a,b). Furthermore, DNA genotyping studies using microsatellite markers from several chromosomes demonstrated that this mole was androgenetic and had arisen by dispermy (Figure 3a,b). We thus considered two possible explanations for the the paradoxical expression of $\mathrm{p} 57^{\mathrm{KIP} 2}$ in the presumptive absence of a maternal genome: (1) loss of the paternal epigenotype at this locus during the ontogenesis of this lesion (via an unknown mechanism), and (2) retention of a maternal chromosome 11, or a fragment thereof encompassing the CDKN1C locus.

To distinguish between these possibilities, 17 microsatellite markers spanning chromosome 11 were compared in maternal and molar tissue. The molar tissue was homozygous for four of the markers and heterozygous for 13. There was no evidence that the molar tissue was androgenetic for chromosome 11. At least one allele at each locus in the molar tissue was consistent with having been maternally derived for all markers. For 11 of the 13 heterozygous markers nonmaternal alleles could also be identified suggesting that the complete mole contained chromosome 11 material from both parents. One of the heterozygous markers was clearly trisomic in the molar tissue, having three different alleles (Figure 3c), while eight other alleles showed clear allelic imbalance consistent with trisomy (data not shown). Although paternal DNA was unavailable for confirmation, these results were consistent with the presence of a maternally derived chromosome 11 in addition to two paternal copies.

To confirm trisomy for the $C D K N 1 C$ region, we performed FISH on tissue sections using as a probe a BAC encompassing the entire CDKN1C locus on 11p. Three distinct nuclear signals were observed in most villous cells (Figure 2c) with no evidence of mosaicism, consistent with the above results. To confirm that the entire chromosome 11 was retained, we performed FISH using centromeric and $\mathrm{q}$ arm probes. Both probes resulted in three signals in most nuclei, consistent with trisomy for the entire chromosome 11 (Figure 2d). We conclude that the 

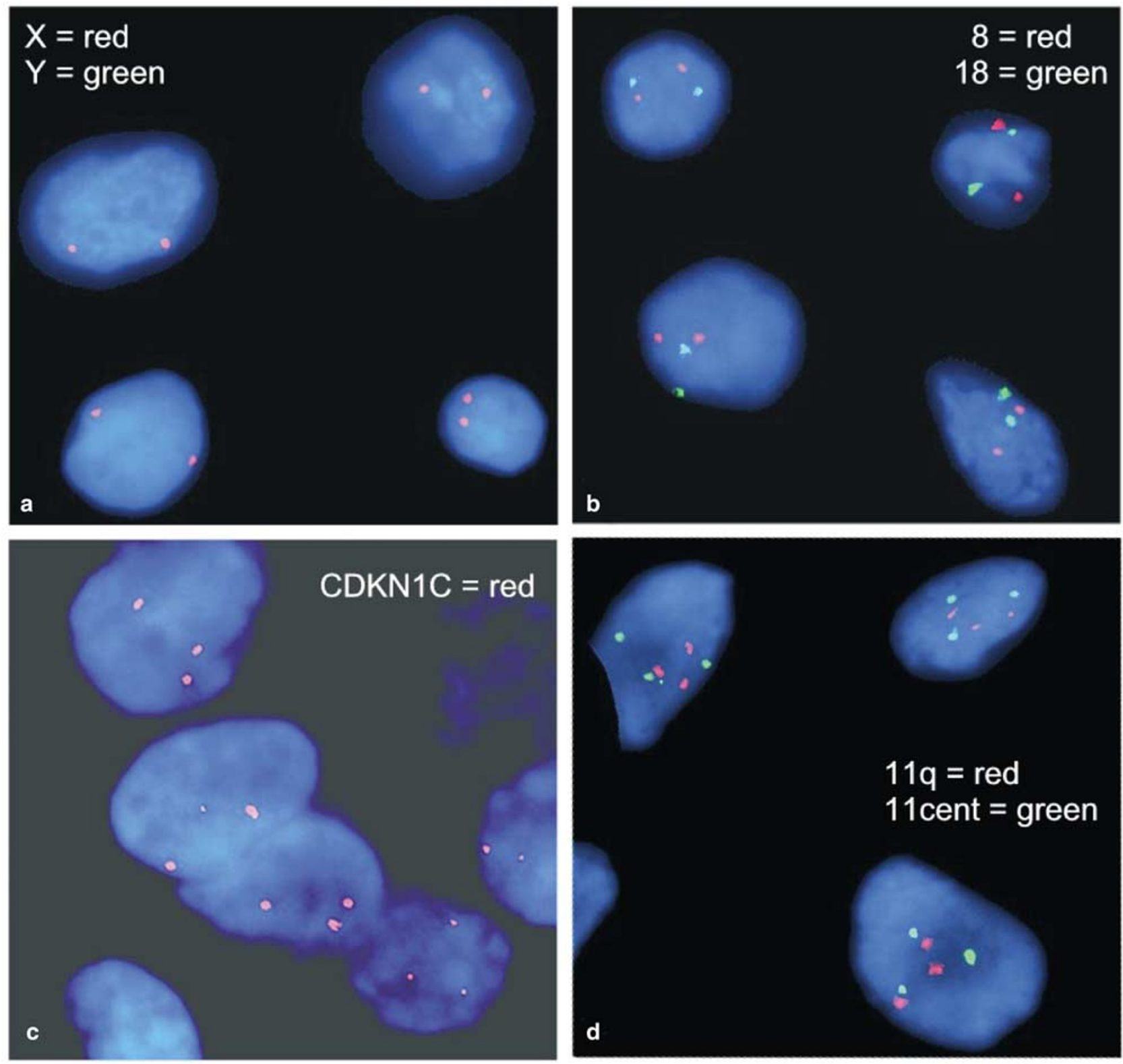

Figure 2 Ploidy studies by FISH, using differentially labeled probes, showing XX diploidy and trisomy 11. Studies were performed on $5 \mu \mathrm{m}$ sections (c) or intact interphase nuclei $(\mathbf{a}, \mathbf{b}, \mathbf{d})$. (a) Cells show two X chromosome signals and no Y chromosome signals consistent with an XX gestation. (b) Villous cells show two signals for both chromosomes 8 and 18 consistent with diploidy. (c) Cells show three signals for probe spanning the CDKN1C locus on chromosome 11p. (d) Cells also show three signals for chromome 11q and centromeric probes.

basis of aberrant expression of both p57 $7^{\mathrm{KIP} 2}$ and PHLDA2 in this complete mole was the retention of a maternal chromosome 11 containing the imprinted gene cluster encompassing the $C D K N 1 C$ and PHLDA2 loci.

\section{Discussion}

We have identified a case of an androgenetic complete mole that, in contrast to other complete moles, shows positive staining of the maternally expressed gene products $\mathrm{p}^{\mathrm{KIP} 2}$ and PHLDA2. We note that this complete mole is dispermic, rather than of the more common monospermic origin. However, this does not account for differences in p5 $7^{\mathrm{KIP} 2}$ and IPL staining, since we have previously shown that neither monospermic or dispermic complete moles usually express p57 ${ }^{\mathrm{KIP} 2}{ }^{4}$ In this unusual case, we have shown that expression of $\mathrm{p} 57^{\mathrm{KIP} 2}$ and PHLDA2 is a consequence of trisomy for the $11 \mathrm{p} 15.5$ region resulting from retention of a maternal chromosome 11 in addition to two paternal copies.

There is insufficient information from this single case to propose a definitive model for the retention 

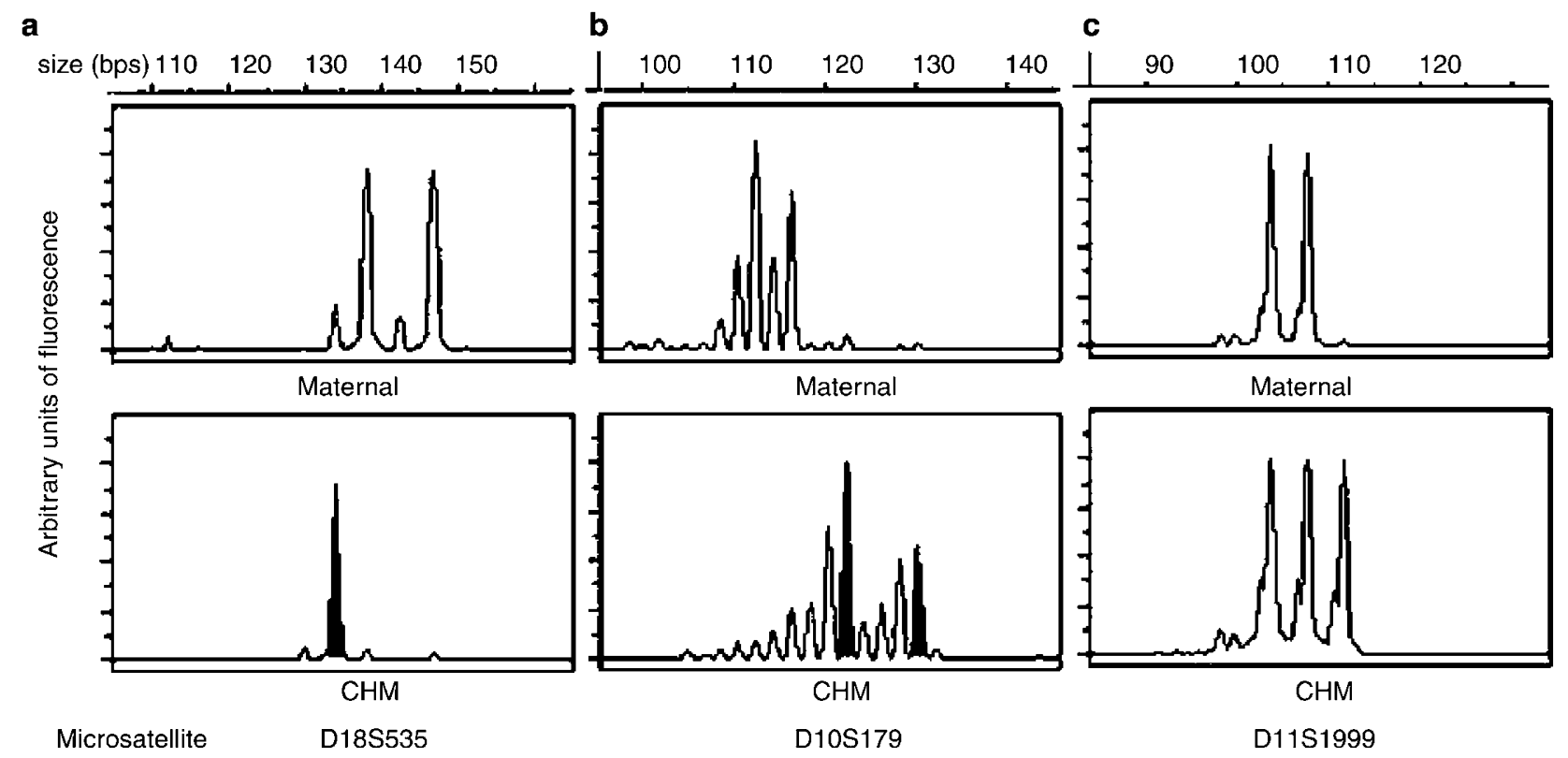

Figure 3 Microsatellite marker analysis in the unusual p5 $7^{\mathrm{KI} 2}$ positive complete mole. (a-c) Microsatellite polymorphisms identified following amplification of DNA from maternal decidua and molar villi with the microsatellite markers D18S535, D10S179 and D11S1999. (a) The complete mole is homozygous for a 134 bp nonmaternal allele (shaded) and therefore androgenetic. (b) The complete mole is dispermic in origin having two different nonmaternal alleles (shaded). (c) The complete mole is trisomic for D11S1999, having at least one nonmaternal allele.

of a maternal chromosome in complete moles, particularly since the mechanisms underlying the loss of maternal genetic material in complete moles are not understood. It can be surmised, however, that the retained chromosome 11 is stably inherited after each cell division, since three copies of chromosome 11 were detectable by FISH in villous nuclei throughout the molar tissue. In contrast, cells of maternal origin (decidua) were uniformly diploid. Although retention of other fragments of maternal DNA in this complete mole cannot be excluded, examination of markers on several other chromosomes $(1,5,7,8,9,10,15,18, \mathrm{X})$, using microsatellite genotyping or FISH, did not reveal any evidence of trisomy for other chromosomal regions.

In this complete mole, p57 ${ }^{\mathrm{KIP} 2}$ and PHLDA2 expression was uniform throughout all molar villi. In addition, FISH demonstrated that all villi were trisomic for chromosome 11. These findings suggest that absence of a maternal copy of the chromosome $11 \mathrm{p}$ imprinted gene cluster, and consequent loss of expression of the maternally transcribed genes in this region is not essential for the genesis of complete moles. However, we cannot exclude the possibility that one or more genes in this cluster is mutated or otherwise dysfunctional, despite the apparently normal pattern and levels of expression of $\mathrm{p} 57^{\mathrm{KIP} 2}$ and PHLDA2.

Our finding of a complete mole that is predominantly androgenetic but which retains a maternal chromosome is the first report of this phenomenon, as far as we are aware. The frequency of this phenomenon-the persistence of DNA of maternal origin from chromosome 11 or other regions of the genome-remains uncertain. By performing immunohistochemistry for $\mathrm{p} 57^{\mathrm{KIP} 2}$ in large numbers of complete moles, we have in effect specifically screened for retention of maternal genome segments encompassing the $C D K N 1 C$ region. We have observed $\mathrm{p} 57^{\mathrm{KIP} 2}$ expression in only this one case out of over 100 complete moles on which we have performed p57 ${ }^{\mathrm{KIP} 2}$ immunohistochemistry as part of research studies on archival material or in the course of diagnostic workup in clinical specimens, suggesting that retention of specific chromosomes is rare. However, aneuploidy of undefined parental origin has been previously described in cytogenetic investigations of complete moles. ${ }^{12-14}$ The overall incidence of retention of maternal DNA in complete moles may therefore be much more common than the retention of chromosome 11 specifically.

p57 ${ }^{\text {KIP2 }}$ immunohistochemistry has become a useful adjunct test in the diagnosis of hydatidiform moles. This study suggests that retention of maternal chromosome 11, or conceivably, a fragment thereof encompassing $C D K N 1 C$, may prove a general mechanism underlying rare false negatives (complete moles that are p57 ${ }^{\mathrm{KIP} 2}$ positive, thereby potentially leading to misdiagnosis as a noncomplete mole). More recently, PHLDA2 immunohistochemistry has also been shown of potential utility in the identification of complete moles. ${ }^{15}$ However, the PHLDA2 locus maps to the same chromosome 11 imprinted gene domain as $C D K N 1 C$, and as expected, PHLDA2 was also strongly expressed in this case due to the presence of a maternal chromosome 11. Additional 
studies will be required to establish the frequency of such false negatives, but our studies suggest they will prove rare ( $<1 \%$ of complete moles). Retention of DNA of maternal origin might contribute to differences in the biological and clinical behavior of complete moles. Identification of additional cases of complete moles retaining maternal DNA (via p57 ${ }^{\mathrm{KIP} 2}$ immunohistochemistry or other methodologies) and further delineation of such cases are needed to shed light on these issues.

\section{Acknowledgements}

RAF is funded by the Cancer Treatment and Research Trust. DHC is supported by a K08 award from the National Cancer Institute of the National Institutes of Health and a Kimmel Scholar Award from the Sydney Kimmel Foundation for Cancer Research.

\section{References}

1 Redline RW, Abdul-Karim FW. Pathology of gestational trophoblastic disease. Semin Oncol 1995;22:96-108.

2 Zaragoza MV, Surti U, Redline RW, et al. Parental origin and phenotype of triploidy in spontaneous abortions: predominance of diandry and association with the partial hydatidiform mole. Am J Hum Genet 2000;66:1807-1820.

3 Castrillon DH, Sun D, Weremowicz S, et al. Discrimination of complete hydatidiform mole from its mimics by immunohistochemistry of the paternally imprinted gene product $\mathrm{p} 57^{\mathrm{KIP} 2}$. Am J Surg Pathol 2001;25: 1225-1230.

4 Fisher RA, Hodges MD, Rees HC, et al. The maternally transcribed gene p57 ${ }^{\mathrm{KIP} 2}$ (CDNK1C) is abnormally expressed in both androgenetic and biparental complete hydatidiform moles. Hum Mol Genet 2002;11:1-6.
5 Nucci MR, Castrillon DH, Bai H, et al. Biomarkers in diagnostic obstetric and gynecologic pathology: a review. Adv Anat Pathol 2003;10:55-68.

6 Frank D, Mendelsohn CL, Ciccone E, et al. A novel pleckstrin homology-related gene family defined by Ipl/Tssc3, TDAG51, and Tih1: tissue-specific expression, chromosomal location, and parental imprinting. Mamm Genome 1999;10:1150-1159.

7 Giannoukakis N, Deal C, Paquette J, et al. Parental genomic imprinting of the human IGF2 gene. Nat Genet 1993;4:98-101.

8 Matsuoka S, Thompson JS, Edwards MC, et al. Imprinting of the gene encoding a human cyclindependent kinase inhibitor, p57KIP2, on chromosome 11p15. Proc Natl Acad Sci USA 1996;93:3026-3030.

9 Saxena A, Frank D, Panichkul P, et al. The product of the imprinted gene IPL marks human villous cytotrophoblast and is lost in complete hydatidiform mole. Placenta 2003;24:835-842.

10 Fisher RA, Khatoon R, Paradinas FJ, et al. Repetitive complete hydatidiform mole can be biparental in origin and either male or female. Hum Reprod 2002;15:594-598.

11 Kuchinka BD, Kalousek DK, Lomax BL, et al. Interphase cytogenetic analysis of single cell suspensions prepared from previously formalin-fixed and paraffinembedded tissues. Mod Pathol 1995;8:183-186.

12 Jacobs PA, Wilson CM, Sprenkle JA, et al. Mechanism of origin of complete hydatidiform moles. Nature 1980; 286:714-716.

13 Yang YH, Kwak HM, Park TK, et al. Comparative cytogenetic and clinicopathologic studies on gestational trophoblastic neoplasia, especially hydatidiform mole. Yonsei Med J 1986;27:250-260.

14 Vejerslev LO, Fisher RA, Surti U, et al. Hydatidiform mole: cytogenetically unusual cases and their implications for the present classification. Am J Obstet Gynecol 1987;157:180-184.

15 Thaker HM, Berlin A, Tycko B, et al. Immunohistochemistry for the imprinted gene product IPL/PHLDA2 facilitates the differential diagnosis of complete hydatidiform mole. J Reprod Med 2004;49 (in press). 